\title{
Development of an optimal control system with pulse control in the rotor circuit for cargo movement mechanisms
}

\author{
Alexey Sinyukov ${ }^{1}$, Tatyana Sinyukova ${ }^{2, *}$, Victor Meshcheryakov ${ }^{3}$, and Elena Gracheva ${ }^{4}$ \\ ${ }^{1}$ Postgraduate, Electric Drive Department, Lipetsk State Technical University, Lipetsk, Russia \\ ${ }^{2}$ Candidate of Sciences, Electric Drive Department, Lipetsk State Technical University, Lipetsk, Russia \\ ${ }^{3}$ Ph.D, Electric Drive Department, Lipetsk State Technical University, Lipetsk, Russia \\ ${ }^{4} \mathrm{Ph} . \mathrm{D}$, Power supply of industrial enterprises, Kazan State Power Engineering University, Kazan, Russia
}

\begin{abstract}
The paper analyzes the methods of starting an asynchronous motor with a phase rotor. The control system used on the object under consideration, the mechanism of cargo movement, is obsolete. Current problems in modern production are measures aimed at energy saving at all stages of production, as well as ensuring the necessary dynamic indicators of control systems. In this paper, several control systems for the mechanism of cargo movement are considered and investigated. The optimal control system for several indicators is proposed. The Matlab Simulink package simulates various start-up methods, namely: direct start-up from the network, rheostatic start-up of an asynchronous motor with a phase rotor, pulse start-up of an asynchronous motor with a phase rotor, asynchronous-valve stage and asynchronous-valve stage with pulse regulation. According to the obtained graphs, a comparison is made and conclusions are drawn about the advantages and disadvantages of each of the start-up methods, as well as graphs of the consumption of active and reactive energy of each of the methods and the most energy-efficient control method is identified.
\end{abstract}

\section{Introduction}

At the enterprises of the metallurgical cycle, mechanisms for moving loads with asynchronous motors with a phase rotor remain involved. The use of such types of motors is relevant in cases where the masses driven are significant, in this regard, the heat generated in the secondary circuit of the engine would cause unacceptable heating of the rotor windings of a shortcircuited motor, as well as at high static torque values. Complete replacement of obsolete control systems containing resistor control systems is not always economically feasible [1-4].

The mechanism under consideration is involved in the metallurgical enterprise. The facility has a $6000 \mathrm{~V}$ motor with a power of $315 \mathrm{~kW}$. The speed of the load movement mechanism [5-7] is regulated by changing the resistance indicators in the rotary circuit (rheostat startup).

With this method of speed control, the starting torque values reach $9900 \mathrm{~N} / \mathrm{m}$, the engine accelerates in 1.625 seconds.

Good adjustment properties are provided by the use of the classical pulse control system [8, 9]. These systems have a relatively low cost.

Energy saving and improvement of the engine performance [10-13] in the control system of an asynchronous motor with a phase rotor is achieved by using an asynchronous valve stage, but a significant disadvantage of these systems is the low power factor, low operating accuracy and high speed.

In this paper, a modified pulse control system is proposed, which combines the positive properties of the classical pulse control systems and the asynchronous valve stage.

A distinctive feature of this system is the presence of a pulse regulator in the rectified current circuit of the rotor, presented in the form of a step-up converter and an inverter, which, unlike an asynchronous valve stage, operates with a constant minimum inverting angle. This ensures a high power factor of the drive in a wide sliding range of the asynchronous motor, and also makes it possible to rationally select the parameters of the equipment from the desired efficiency, depending on the operating mode of the pulse controller.

The use of cascade circuits with pulse control allows you to increase the power factor of the drive, expand the control range, reduce weight and dimensions. It is especially advisable to use pulse control in the case of several regulated motors, while it is possible to use one common inverter, since the motor speed is controlled by a controlled thyristor switch in the rectified current circuit of the rotor $[14,15]$.

\footnotetext{
* Corresponding author: stw0411@,mail.ru
} 


\section{Methods}

\subsection{Pulse speed control system}

The pulse speed control is based on a smooth change in the additional resistance in the rotary circuit by changing the relative time of the conducting state of the transistor switch, which performs the functions of a pulse-width converter. The model of the proposed control system in Matlab is shown in Fig. 1. In the rotor circuit there is a three-phase uncontrolled rectifier VD, which serves to rectify the three-phase voltage of the rotor to a constant voltage. In the DC link, the choke $\mathrm{L}$ is installed in series, which is necessary to smooth out the ripples of the rectified current of the rotor. Next, a power IGBT transistor is installed and a shunt resistance $\mathrm{R}=35 \mathrm{ohms}$ is parallel to it. This is the total resistance of the stages during rheostatic start-up.

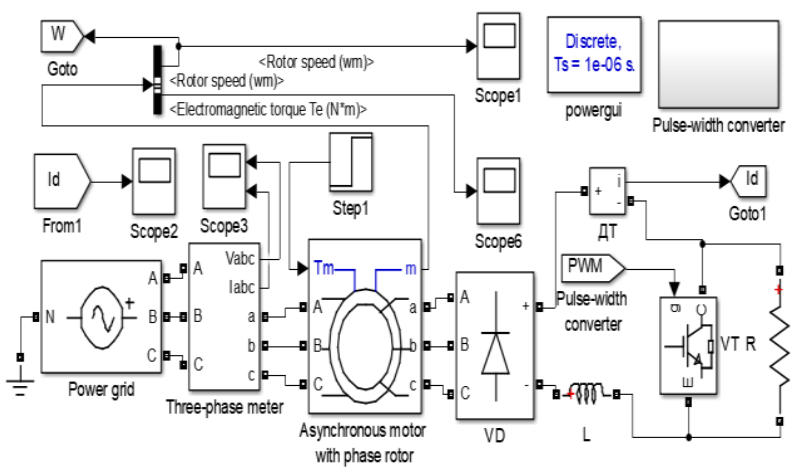

Fig. 1. Model of a system with pulse speed control.

The implementation of the "Pulse-width converter" block is shown in Fig. 2.

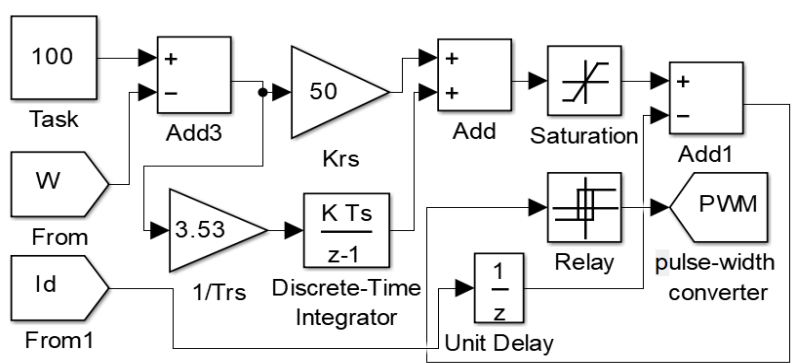

Fig. 2. Block «Pulse-width converter».

The pulse-width conversion system consists of a task for the drive speed, in this model $100 \%$ is set, a speed controller PI, a current setting unit that helps to keep the set current value (200A was set) in the DC link as the drive accelerates. After the current setting block, the signal is sent to the relay current controller, from which the task for the conducting state of the key, called the duty cycle, is applied. The duty cycle forms a pulse switching of the resistance in the rectified current circuit of the rotor.

\subsection{System with asynchronous-valve stage with pulse regulation}

A feature of the electric drive operating according to the scheme of the asynchronous-valve stage is the presence in the system of an asynchronous motor with a phase rotor and a frequency converter with a DC link. This scheme allows for contactless smooth start - up and motor speed control, by changing the sliding value of the motor rotor by introducing a controlled counter-EMF value into the rotor circuit using a frequency converter. The effect on the counter-EMF of the rotor is carried out by changing the opening angle of the power keys of the inverter according to a given law.

The model of the asynchronous-valve stage with pulse speed control is shown in Fig. 3.

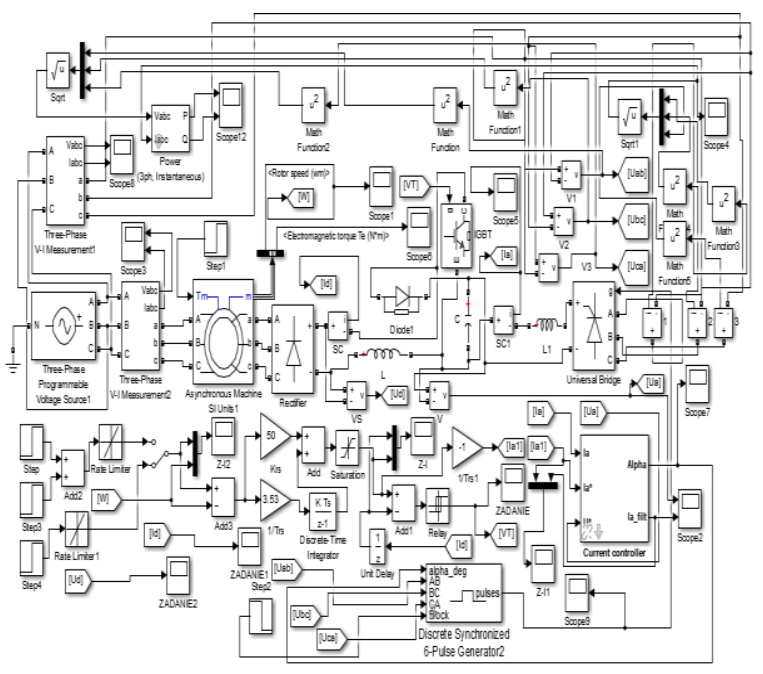

Fig. 3. Model of asynchronous valve stage with pulse regulation.

A three-phase bridge uncontrolled rectifier is included in the AC circuit of the rotor. To smooth out the ripples of the rectified current of the rotor, a choke $\mathrm{L}$ is installed. A power IGBT transistor is connected in series to the choke on the positive $(+)$ and negative (-) terminals of the rectifier, for shunting the DC link with a certain duty cycle, as the drive accelerates. The power transistor is controlled by pulse-width modulation based on a relay current regulator (Fig. 4). The difference between pulse-width modulation and pulse-width conversion is that it is possible to form a speed task with a linear increase using an intensity setter, which allows you to change the acceleration time of the drive to the nominal speed.

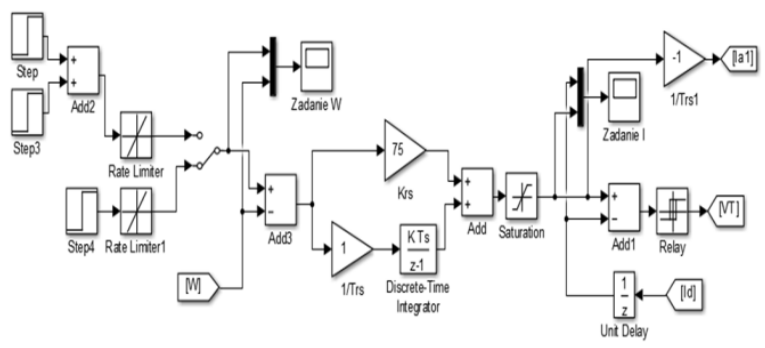

Fig. 4. Pulse-width modulation unit. 
Also in this model, it is possible to track the generated signal and the task signal.

In parallel to the power IGBT transistor, a capacitor $\mathrm{C}$ is installed. To charge the capacitor, a diode is built in series with the power switch, turned on opposite to the capacitor, it is necessary for the capacitor to discharge in the direction of the thyristor inverter. Between the capacitor and the inverter, a choke L1 is connected, which has the same purpose as the choke $\mathrm{L}$. The inverter is switched on in reverse, relative to the three-phase unmanaged bridge rectifier installed in the rotor circuit.

The pulse-phase control system of the inverter (Fig. 5) consists of a PI-current regulator with a voltage warning. Based on this voltage, the angle for the formation of pulses arriving at the thyristors in a threephase inverter is recalculated according to the formula of the adjustment characteristic.

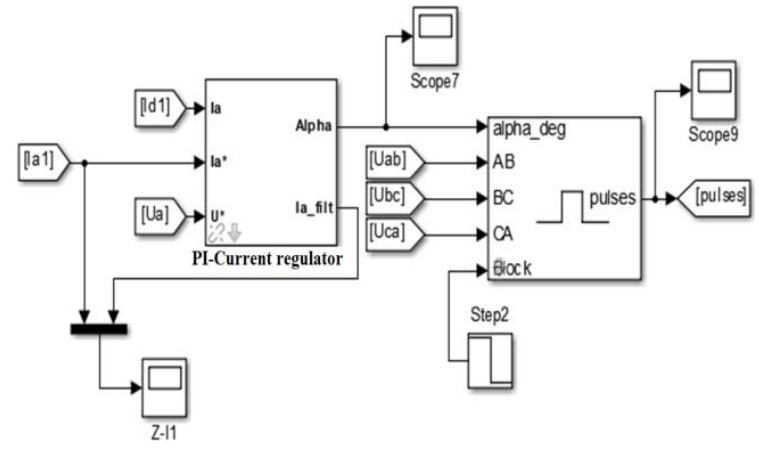

Fig. 5. Inverter pulse-phase control system.

The current regulator uses a Low-pass filter-a frequency filter to filter out the pulsating component of the current (Fig. 6).

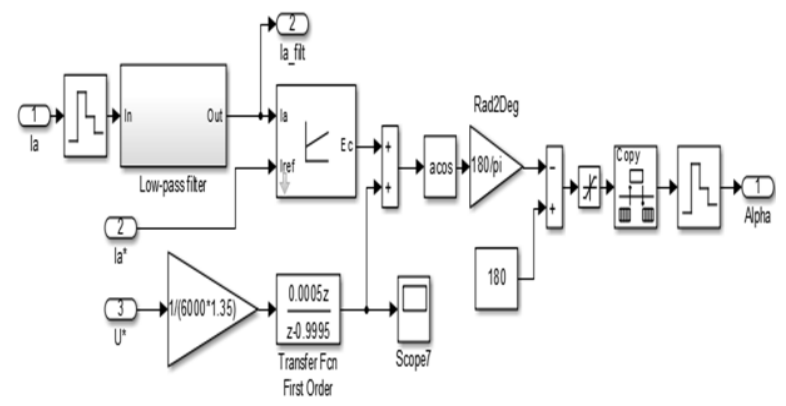

Fig. 6. Structure of the PI current regulator.

The task for forming the opening angle of the thyristors is fed to a discrete synchronized 6-pulse generator, at the output of which we have pulses for controlling a three-phase bridge thyristor inverter.

After inverting, the alternating current enters the network.

\section{Simulation results}

\subsection{Pulse speed control system}

Fig. 7 and Fig. 8 show combined graphs of the transient processes of torque and speed when starting the drive without load with rheostat and pulse control methods.

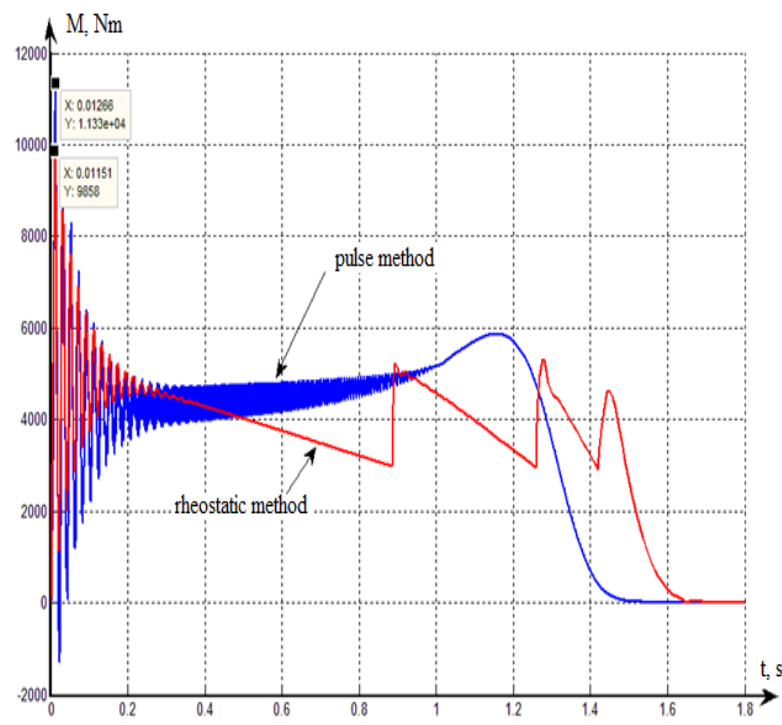

Fig. 7. The transition process of the moment.

According to the transient process of moments, we can observe that the starting moment in the pulsed control method is greater than in the rheostatic control, which gives advantages in dynamics, but the moment has a pulsating character until it approaches the critical moment, since the rectified current of the rotor is stabilized. It can also be seen that with the pulse control method, the drive accelerates faster.

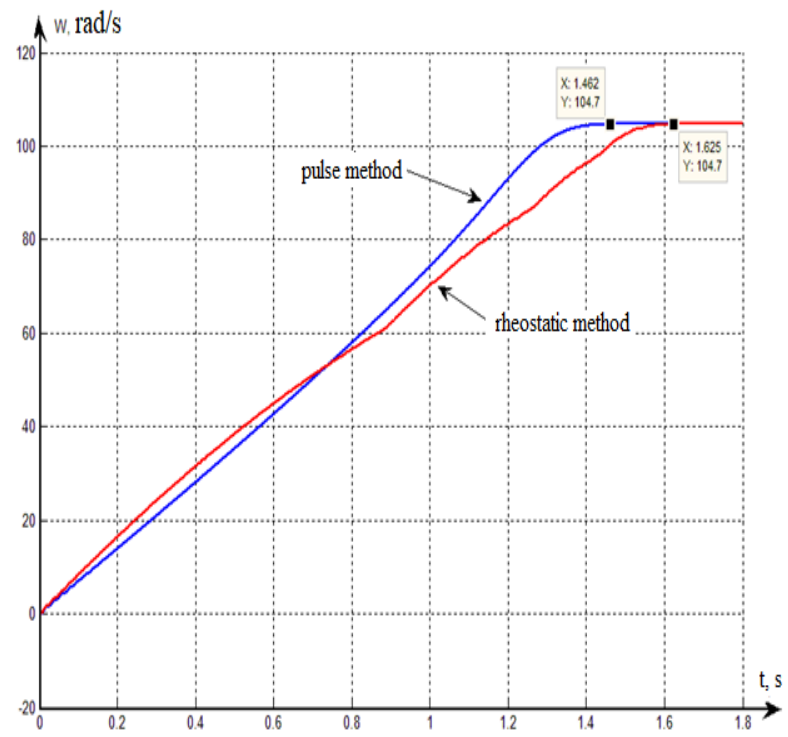

Fig. 8. Speed Transition.

According to the speed graph, it can be seen that with pulse control, the speed is formed faster and has a more linear character than the speed with rheostatic start-up, where the drive accelerates unevenly, which is critical for cargo movement mechanisms.

Fig. 9 shows a combined graph of the moment transients in the pulsed control method and in the rheostatic control method under load. The rated motor torque of $3167 \mathrm{~N} / \mathrm{m}$ was set as the load. 


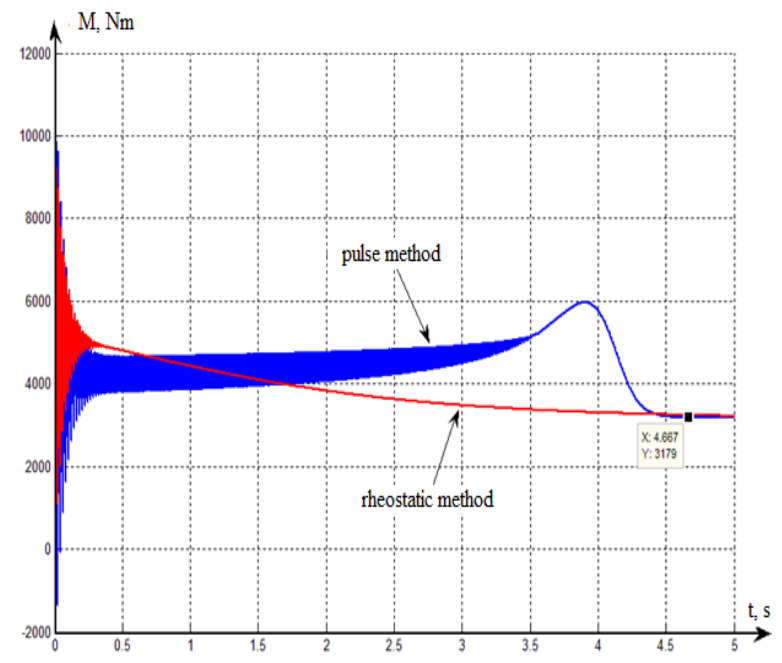

Fig. 9. The transient process of the moment in the pulsed and rheostatic method of regulation under load.

The graph shows that the start-up under load by the pulse method is carried out for a longer time than without load, for 4.68 seconds, after which the drive reaches its nominal speed. The load is applied at the initial time. However, the graph of the rheostatic start-up method shows that the drive did not start at all, since the rheostatic start-up method takes place only if the drive is already started and the load is applied to the working motor. This leads to the conclusion that the pulse method has greater dynamics and can accelerate under the load applied at the initial moment of time, and a greater starting torque than the rheostatic starting method.

\subsection{System with asynchronous-valve stage with pulse regulation}

The opening angle of the thyristors (Fig. 10) was set to $\alpha=160^{\circ}$, since the three-phase thyristor bridge operates in the inverter mode.

The value of the angle decreases as the drive accelerates, since the voltage in the DC link of the asynchronous-valve stage with pulse regulation decreases in proportion to the slip.

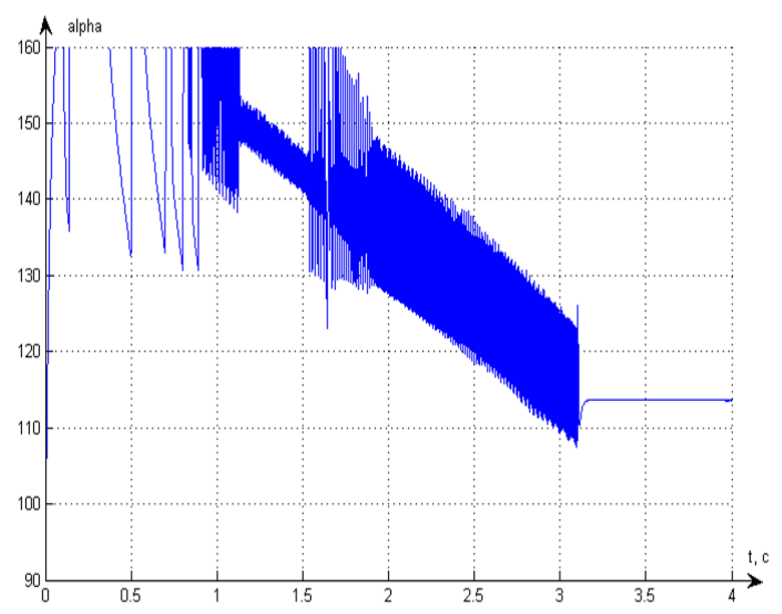

Fig. 10. Creating a task for the opening angle of thyristors.
The transient torque process is shown in Fig. 11. The start was made under load, where the rated torque of the engine was used as the load.

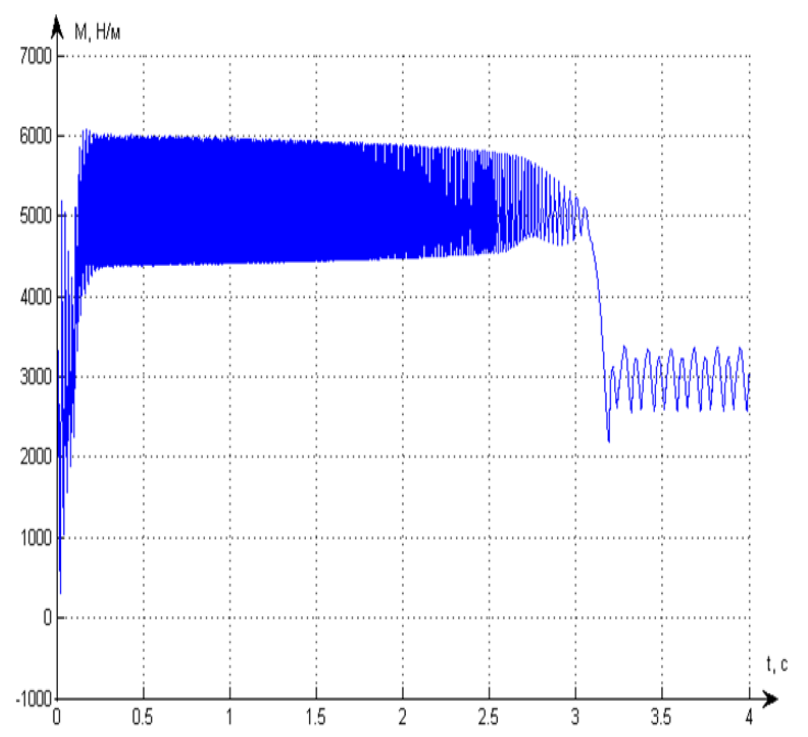

Fig. 11. The transient process of the moment under load.

Under load, the drive starts up in the same time as without load. The moment under load after reaching the rated speed has a pulsating character.

\section{Discussion}

Comparison of energy efficiency indicators of the proposed speed control methods is possible on the basis of active power data (Fig. 12) and energy consumption indicators (Fig. 13) during the start-up period. The results obtained in the simulation were compared with the data obtained in the simulation of the currently available control system on the mechanism under consideration and with the indicators of direct start of an asynchronous motor with a short-circuited rotor.

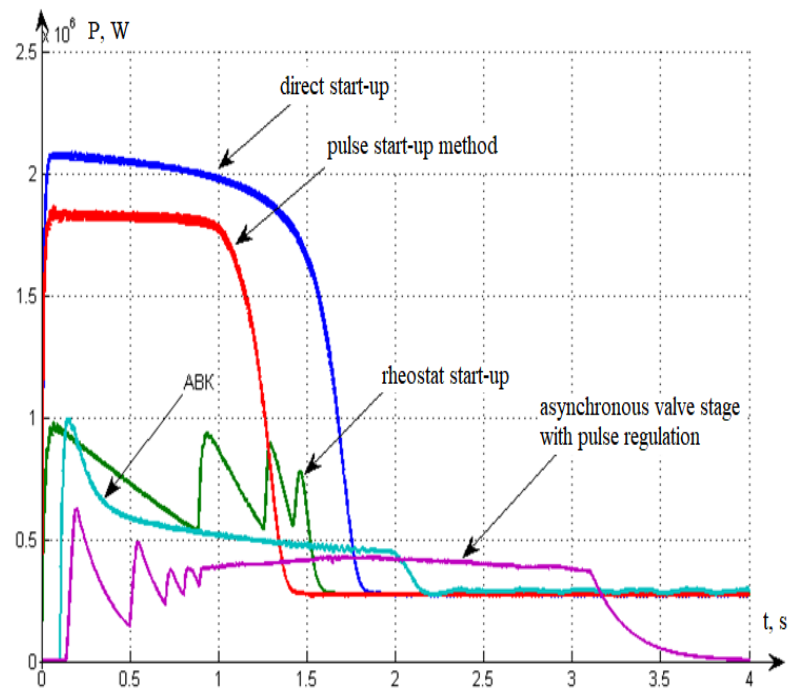

Fig. 12. Active power consumption schedule $P$. 


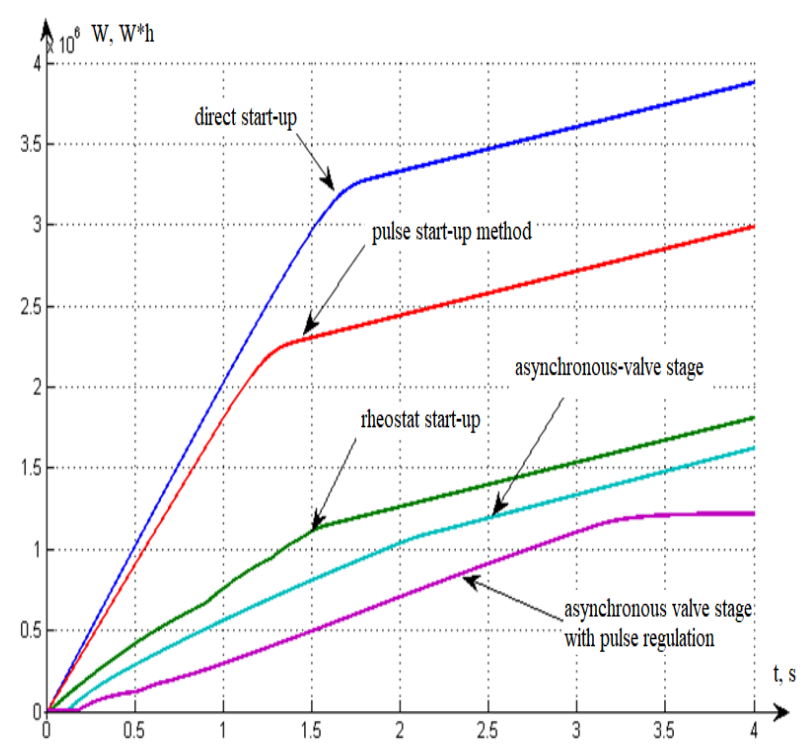

Fig. 13. Schedule of energy consumption during start-up.

Based on the obtained data, it can be concluded that the most energy-efficient method is an asynchronousvalve stage with pulse control in the rotor circuit.

\section{Conclusions}

Based on the conducted research, the following conclusions can be drawn:

- the use of the pulse method of speed control allows you to start the engine of the load movement mechanism under load;

- the use of the pulse method allows you to increase the smoothness and rate of formation of the speed characteristic;

- the proposed method of speed control, due to the use of a combined system, is the most energy-efficient.

\section{References}

1. Mbika Muteba, Mamakhetha Eveline Makhetha. Impact Analysis of Number of Broken Rotor Bars and Rotor Bar Shape Types through Illation Method for Asynchronous Motors. 2019 11th International Conference on Electrical and Electronics Engineering (ELECO), 2019, 19359523, November 28-30

2. A. M. Ziuzev, V. P. Metelkov. On heating induction motor rotor at start-up of high-inertia mechanisms. 2018 17th International Ural Conference on AC Electric Drives (ACED), 2018, 17719968, March 26-30

3. Yongming $\mathrm{Xu}$, Mengmeng Ai, Dajun Tao. Investigation of the heat characteristic based on the exergy destruction rate for high power density motor. 2017 20th International Conference on Electrical Machines and Systems (ICEMS), 2018, 17239986, August 11-14

4. Watanabe N., Hirotsuka I., Sugimoto H., Nakamura M. Trial Fixed Outer Rotor Three-Phase Squirrel-
Cage Induction Motor and its Basic Characteristics. 2018 21st International Conference on Electrical Machines and Systems (ICEMS). Jeju, Korea (South). 2018. Pp. 135

5. Nikolai Karnaukhov, Maxim Filimonov, Dmitry Smyatsky, Roman Mironenko. Improving The Motion Parameters Of The Production Mechanism Link By Microvibration. 2020 27th International Workshop on Electric Drives: MPEI Department of Electric Drives 90th Anniversary (IWED), 2020, 19534832, January 27-30

6. T.V. Sinykova, E.V. Sentsov and A.V. Sinyukov. Neural Network Speed Observers. Proceedings 2019 1st International Conference on Control Systems, Mathematical Modelling, Automation and Energy Efficiency (SUMMA),Lipetsk, Russia , 288 (2019)

7. V. Meshcheryakov, T. Sinyukova, A. Sinyukov, and O. Vladimirov. Analysis of the effectiveness of using state observers in the control system of the traveling mechanism of the bridge crane trolley. E3S Web of Conferences. Sustainable Energy Systems: Innovative Perspectives (SES-2020), Saint-Petersburg, Russia, 2020, 220, 01084, October 29-30

8. Shavkat Ismatovich Fayziev. Synthesis of an Automatic Control System with Pulse-width Modulation According to the Speed Criterion. 2020 International Conference on Information Science and Communications Technologies (ICISCT), 2020, 20389516, November 4-6

9. Zhi-Wei Liu, Xinghuo Yu, Zhi-Hong Guan, Bin $\mathrm{Hu}$, Chaojie Li. Pulse-Modulated Intermittent Control in Consensus of Multiagent Systems. IEEE Transactions on Systems, Man, and Cybernetics: Systems, 2017, 16808579, Volume: 47, Issue: 5, May 2017

10. Y.I. GrachevaY.I. Gracheva, N.A. Alimova. Calculating Methods and Comparative Analysis of Losses of Active and Electric Energy in Low Voltage Devices. International Ural Conference on Electrical Power Engineering (UralCon), 2019, pp. 361-367 (2019)

11. V. Meshcheryakov, T. Sinyukova, A. Sinyukov, and O. Vladimirov. Analysis of the effectiveness of using the block for limiting the vibrations of the load on the mechanism of movement of the bogie with various control systems. E3S Web of Conferences. Sustainable Energy Systems: Innovative Perspectives (SES-2020), SaintPetersburg, Russia, 2020, 220, 01059, October 2930

12. Jiang You, Minghao Liu, Jiarui Ma, Hongjie Jia Modeling and Analyse of Induction Motor Drive System with Consideration of DC Bus Stabilization and Control Performance. 8th International Power Electronics and Motion Control Conference (IPEMC 2016 - ECCE Asia). 2016. Pp. 1362-1368

13. Y.I. Gracheva, N.V Chernova, A.I Fedotov, E.A. Fedotov, Local Fourier transformation application 
for mathematic modeling of synchronous machine valve actuator. Journal of engineering and applied sciences 11, 1, 2939-2945, 2016

14. Mehmet Fatih Cankurtaran, Ali Eren Kocamis. Sensorless Speed Control of PMDC Motor with Cascade PI Controller. 2019 International Symposium ELMAR. Zadar, Croatia. 19240666, 2019.

15. Atmanandmaya, Jayaram Nakka. Single Phase Symmetrical Nine level Cascaded Multilevel Inverter by Single Carrier PWM Scheme. 2018 IEEE International Students' Conference on Electrical, Electronics and Computer Science (SCEECS). Bhopal, India. 18302749, 2018. 